\title{
A MODEL FOR SPONTANEOUS MUTATION IN DROSOPHILA CAUSED BY TRANSPOSING ELEMENTS
}

\author{
JAMES N. THOMPSON, JR.* and R. C. WOODRUFF' \\ * Department of Zoology, University of Oklahoma, Norman, Oklahoma U.S.A. 73019; \\ † Department of Biological Sciences, Bowling Green State University, \\ Bowling Green, Ohio 43403
}

Received 2.iv.81

\section{SUMMARY}

\begin{abstract}
Transposing genetic elements make up a significant proportion of the DNA of at least some eukaryotes. One of the potential side effects of transposition is a high rate of apparently spontaneous mutation. In this paper we consider some of the evolutionary strategies that might be involved in the suppression of transposition, and we outline a model for the control of spontaneous mutation in terms of the regulation of transposition. Specific predictions are based upon the well-characterized genetics of the mutator system associated with hybrid dysgenesis in Drosophila melanogaster, which has many parallels with the expected behaviour of transposing elements.
\end{abstract}

\section{INTRODUCTION}

IN its most general formulation, evolution is a change in gene frequencies. Recently, however, the classic view that gene frequencies are altered by mechanisms such as natural selection of adaptively favoured phenotypes has been challenged, at least in so far as it applies to certain classes of apparently non-coding DNA (Doolittle and Sapienza, 1980; Orgel and Crick, 1980; Orgel et al., 1980; Dover and Doolittle, 1980). The hypothesis is that classes of DNA can arise whose only "function" is self-preservation. In the absence of any direct influence upon the organism's phenotype, the only selection upon them would be through intracellular conditions affecting their individual survival. These, then, might be thought of as the ultimate "selfish genes" (Dawkins, 1976; Crick, 1979).

Geneticists seem indoctrinated with the idea that selection has to act through the gene's expression in an individual phenotype. On the other hand, prokaryotic transposable elements and eukaryotic middle-repetitive sequences do not seem to fit into this idea of having a phenotypic influence. While an explanation in terms of "non-phenotypic selection" (Doolittle and Sapienza, 1980; Orgel and Crick, 1980) is attractive in many ways, not the least of which is its simplicity, a slightly different perspective also makes interesting predictions.

One problem seems to be that the typical idea of a phenotype, as broad as it is, may nevertheless be too restricted when applied to certain genetic effects. Some genes do not act upon processes in a way that makes them detectable in the phenotype of a single individual. In particular, "rates", such as mutation rate, must be defined in terms of a group and, thus, become "population phenotypes". This is not necessarily group selection in the traditional sense. From the population point of view, even in the absence of 
a direct phenotypic effect in the carrier, one can visualize genes that have a significant influence upon various rate-dependent components of evolution in a gene pool.

We suggest that eukaryotic middle-repetitive sequences are representatives of this sort of gene. Rather than having no phenotypic effect, these factors may have one of the most far-reaching effects possible-the induction of spontaneous mutation.

\section{NOMAdic DNA AND MUTATION RATES}

At first, induction of spontaneous mutation may seem self-contradictory. It is not. Mutation events are "spontaneous" only because we do not know all of their causes. Environmental influences such as temperature, age, transcription crrors, and mutagenic and infectious agents are well-known, but probably play a comparatively minor role in natural populations (Auerbach, 1976; Woodruff et al., 1982). Much more potent are genetic modifiers which appear to be at least indirectly involved in the evolution of mutation rates.

The hybrid dysgenesis, or "male recombination" (MR), mutator system in Drosophila melanogaster (Thompson and Woodruff, 1978a, 1978b, 1980) has many characteristics one would expect of transposable elements. Hybrid dysgenesis is a collection of genetic phenomena which includes the induction of visible and lethal mutations, male recombination, distortion of segregation, sterility, and other genetic events. These are induced in crosses among strains in Drosophila, and may occur in other organisms as well (Woodruff and Thompson, 1980). In terms of mutation, the two most important similarities with transposable elements are, first, that the $M R$ elements that map at one position can later move to new sites and even to nonhomologous chromosomes (Slatko, 1978), and, second, that $M R$-induced mutations are often unstable, reverting at extraordinarily high frequencies (Golubovsky et al., 1977; Thompson and Woodruff, 1978a, 1978b, 1980; Engels, 1979). In addition, there is site preference in mutant induction (Green, 1978), deletions are commonly produced in association with mutation (Green and Shepherd, 1979), and rearrangements and other chromosome breakage events occur at significant frequencies (Voelker, 1974; Henderson et al., 1978; Yannopoulos, 1978).

The best characterized groups of transposable elements in Drosophila are the dispersed, middle-repetitive sequences represented by copia and at least several dozen other families. These comprise up to 15 per cent (and maybe more) of the Drosophila genome (Young, 1979). Evidence of transposition is of several types. First, different laboratory strains have approximately equal numbers of copia sequences, but they are found at different locations as shown by in situ hybridization (Ananiev et al., 1978; Young, 1979; Strobel et al., 1979). Second, Potter et al. (1979) document the transposition of copia into different sites in the DNA of tissue culture cells. Even the dramatic transposition of the Ising transposon (Ising and Ramel, 1976) may be correlated with copia behaviour (Gehring and Paro, 1980). These observations also appear to apply to other middle-repetitive families (Potter et al., 1980; see also report by Mirzabekov, 1981).

A conservative calculation of the total number of transposing elements indicates the magnitude of potential assault upon genetic integrity in 
Drosophila. An estimated 30 or more families having an average of 30 dispersed members per family (Young, 1979) yields an expectation of about 1000 transposing genetic elements in the genome. Since new families are still being identified and the number of members per family varies widely (Young, 1979), there may be many more than 1000 elements inserting into new positions on the host chromosomes. It is, therefore, reasonable to expect the phenotype "mutation rate" to be affected by their activity.

The prediction, from an evolutionary point of view, is that mutation rate will be minimized, or optimized, by natural selection (Sturtevant, 1937; Ives, 1950; Williams, 1966; Leigh, 1973). Yet, the ways in which this can be accomplished are diverse. For example, if transposable elements were responsible for at least some spontaneous mutation, selection could minimize mutation either by limiting the amount of transposition or by decreasing its deleterious effects. Indirect evidence suggests that movement is fairly common. If movement were rare, one would not expect to find major differences among strains in the sites that are occupied by members of middle repetitive families. Large differences do, however, exist (Ananiev et al., 1978; Young, 1979; Strobel et al., 1979).

The potentially harmful effects of frequent transposition can be decreased in a variety of ways. For example, selection could favour a recognition mechanism that increases the probability that the elements will insert in a neutral, or non-coding, region. There is, in fact, some evidence in favour of this possibility. First, at least one of the sequenced $T y$ elements in the his 4 locus in yeast is in the $5^{\prime}$ non-coding region (Cameron et al., 1979; Roeder et al., 1980). Second, one finds that many hybridizing sites for transposable elements in Drosophila are concentrated in heterochromatin (Young, 1979). These observations do not, of course, imply that such insertions are always phenotypically undetectable. Yet, the effects of such insertions are, in general, likely to be less severe than those within a coding region unless the inserted sequences are spliced out before translation.

On the other hand, it is probably unreasonable to expect all insertions to be in mutationally "neutral" sites. Indeed, examples of mutations containing members of one or more middle-repetitive sequence families are being found more and more frequently (see, for example, the report by Mirzabekov, 1981). If insertional sites are potentially deleterious, selection could favour any of a wide variety of means of regulation, and genetic analysis of the hybrid dysgenesis system in Drosophila melanogaster offers a powerful way to study them.

For example, both mutation and chromosome breakage studies confirm that mutator activity is decreased or totally suppressed within a strain, but it is released from suppression in certain crosses between strains (Woodruff et al., 1979; Thompson and Woodruff, 1980). In addition, while mutator factors can be mapped, the large difference between mutation rates in reciprocal crosses shows that the system also has an extra-chromosomal component. The simplest explanation is that a genotype-cytoplasm interaction minimizes mutation by inhibiting the movement of transposable elements. A precedent for suggesting some type of external control of transposition is provided by $T y 912$ in yeast, in which movement is under the control of unlinked Spm elements (Farabaugh and Fink, 1980; Roeder et al., 1980). McClintock's $A c$ element represses its own transposition 
(McClintock, 1952), and other transposable elements carry repressor genes regulating their own transposase function (Heffron et al., 1979).

Thus, the regulation of mutation could be accomplished by stabilizing transposable elements within a strain or population, fixing both their number and their positions. In such a model, the extra-chromosomal component would probably act upon an element to stabilize it in position (e.g., inhibit a transposase), rather than by blocking open insertion sites. There are several reasons for suggesting this. First, the number of potential insertion sites is extremely large (perhaps unlimited), and direct blocking of such sites would be inefficient and would be likely to interfere with normal gene regulation. In addition, while the processes of insertion and deletion may be independent in prokaryotes, it is necessary to hypothesize some degree of correlation between them in Drosophila in order to account for the apparently constant copy number within each strain (cf. Young, 1979). If a transposon stayed at its original chromosomal position while a copy of itself was added elsewhere, numbers of inserted elements would increase. This has not been observed in the few Drosophila strains that have been examined in sufficient detail. Similarly, if elements could move freely, but open sites were blocked, the number of elements would tend to decrease as they became excised but not reinserted. This possibility is also unsupported by the data.

The balance of evidence is, therefore, upon control of mutation through control of transposition itself. The placement of inserted elements could, however, vary among strains. Indeed, a comparison of the number and position of copia and copia-like hybridization sites in the Oregon R, Canton S, Seto, and Swedish C strains (Strobel et al., 1979) shows the uniformity within, but differences among, strains predicted by this model. In some crosses between different strains, however, this stabilizing system breaks down, elements presumably transpose to new sites, and an increase in mutation is recorded (Woodruff and Thompson, 1980; Thompson and Woodruff, 1978a, 1980).

In addition to differences between reciprocal crosses, one finds that there is significant variation among wild strains in the amount of mutator activity released by outcrossing (Woodruff and Thompson, 1980). We propose that this quantitative variation among strains is due to a difference in the number of elements present in the strains crossed. Let us assume, for example, that a control system acts through the cytoplasm to stabilize the elements carried by a particular strain. The cytoplasmic component (stabilizer) is quantitatively balanced with the genetic elements. No transposition would occur until this balance was upset in crosses to different strains. A "low mutator" line would be one that had comparatively few such elements and a correspondingly low level of cytoplasmic inhibition. Even when this system breaks down in a cross between strains, little if any mutation occurs because there are only a few elements that can transpose. One might expect laboratory lines to be predominantly of this type, since almost any source of variability would be selected against by the researcher or by intense competition in a comparatively homogeneous laboratory culture environment. This expectation fits the available evidence about mutator activity and other components of hybrid dysgenesis in laboratory lines (see reviews in Kidwell et al., 1977; Thompson and Woodruff, 1978a; Woodruff and Thompson, 1980). 
Natural populations could, however, tolerate a much higher potential mutation rate. A larger number of elements could be inserted and stabilized in the genome under the control of a higher level of cytoplasmic inhibition. Under most circumstances, it would still be advantageous for mutation rates to be minimized, but the consequences of a burst of newly-induced mutations would not necessarily be disastrous, given the heterogeneity of natural environments, the value of variation for colonizing new habitats, and large population sizes. Indeed, bursts of mutation have been documented in natural populations (Spencer, 1935; Alexander, 1949; Berg, 1965). While we have generally considered such mutations disruptive to the population, perhaps even contributing to isolation among subpopulations, there is the possibility that some mutations are constructive. Some nomadic elements (e.g., copia) are transcribed, and Williamson et al. (1981) have described the insertion of a transposable element near the $5^{\prime}$ end of the structural gene for yeast alcohol dehydrogenase II that produced constitutive expression of the normally glucose-repressed enzyme. Furthermore, acting individually or in pairs, these elements may cause transpositions of large blocks of DNA (cf. Gehring and Paro, 1980), perhaps moving them to differently regulated regions of the genome.

The model we have outlined here, in which potential mutation rate is a function of the number of transposable elements in the genome, is consistent with several experiments that compare the cytoplasmic effects in crosses of dysgenic lines differing in their levels of mutator activity. In particular, the model accounts for the difference in expressed mutator activity in genotypically similar individuals from reciprocal crosses. Like other dysgenic traits, mutation frequency is higher when the parent male is from a wild type strain (Kidwell et al., 1977; Thompson and Woodruff, 1978 a). According to this model, the parental male of a cross between a wild type male and a laboratory strain female would carry a larger number of transposable elements than would the females. The cytoplasmic contribution in the eggs of females, however, would be low in stabilizing ability. In the progeny of this cross, then, there would be more elements in the genome than could be stabilized by the cytoplasm, unstabilized elements would transpose, and mutations and chromosome breakage would result. In the reciprocal cross, wild type females would contribute a cytoplasm capable of stabilizing more elements than inherited from the laboratory strain male, and no transposition would occur. Such reciprocal differences in mutation are well-documented (e.g., $O K 1$ mutator line males crossed to non-MR Canton $\mathrm{S}$ females $=61 / 2982$ sex-linked visible mutations, $2 \cdot 04$ per cent; Canton $S$ males crossed to $O K 1$ females $=5 / 4342$ mutations, $0 \cdot 12$ per cent; Woodruff and Thompson, 1980).

When expanded to include reciprocal crosses between different natural populations, the level of mutation should be predictable from the level of potential mutator activity in the strains involved (i.e., it should be correlated with the number of transposable elements they carry). Consider, for example, two different wild type strains crossed to the same standard laboratory stock. The mutation frequency in the progeny shows that one (line A) is a high mutator line and the other (line B) has a lower mutation rate, intermediate between $A$ and the laboratory line. Mutation rate is negligible in crosses within each strain $(\mathrm{A} \times \mathrm{A}$, or $\mathrm{B} \times \mathrm{B})$. Many such examples have been reported (Thompson and Woodruff, 1978a, 1980; 
Woodruff and Thompson, 1980). In terms of our model, both lines carry elements of one or more families that are unstabilized in crosses to laboratory lines. When $\mathrm{A}$ is crossed to $\mathrm{B}$, or $\mathrm{B}$ to $\mathrm{A}$, however, the level of released mutator activity would depend upon the difference between strains in the number of transposable elements they carry.

Furthermore, the relative number of elements within each family becomes important if cytoplasmic suppression is different for each family of elements. Evidence that stabilization is family-specific is sparse but includes the fact that, although the number of elements within a particular family is essentially constant among individuals, different families in a strain have different numbers of elements (e.g., about 100 sites for plasmid cDm2029, 33 sites for $\mathrm{cDm} 2046$, and 3 sites for $\mathrm{cDm} 2067$ in one line; Young, 1979).

Indeed, family-specific suppression is precisely what one would expect if these elements arose in, or invaded, the genome at different times in the evolution of the species. Drawing from the parasitic DNA hypothesis of Doolittle and Sapienza (1980) and of Orgel and Crick (1980), one would predict that a novel transposing sequence would increase rapidly until the "host" controlled or suppressed it in some manner. If only a single mechanism were involved, however, one would not expect subsequent sequences to be able to establish themselves. Only those would survive that could circumvent the previous suppression systems, such as by employing a slightly different regulatory mechanism controlling transposases (Shapiro, 1979; Cohen and Shapiro, 1980).

The number of factors "released" to transpose in any particular cross would, therefore, be a function of the family by family differences in number of elements and level of cytoplasmic suppression. Thus, detected mutation and transposition should show a reciprocal cross difference, like that for mutation in crosses to laboratory lines, though in the case of wild populations, it would not be an all-or-none effect. The frequency of mutation would be highest in the progeny of a cross between a high mutator line $(\mathrm{A})$ male and a low (B) female. A lower amount of mutation would also be expected in the reciprocal. Cytoplasmic factors of the high line would not be expected to stabilize all of the families transmitted by the low line, since the size of some families in B would be higher than in A. In fact, this is exactly what is seen in some crosses between mutator lines (Thompson and Woodruff, 1978a, 1980). The most extreme form of this model would be one in which a particular family was present in one line, but not in the other. Such an example would probably show the highest, most readily detected increase in mutation rate.

\section{Conclusions}

The predictions from a genetic analysis of mutator activity associated with hybrid dysgenesis are straightforward, but perhaps somewhat difficult to test. The difficulty resides in the large number of different families of transposable elements that may be present in the genome. Not all families may behave in the same way, and the type and number of members per family may vary greatly. The principal prediction of our model is that the number of transposable elements in a line recently obtained from natural populations will be higher than that found in laboratory stocks. Since 
regulation of each family may differ, however, an extensive survey of families in a variety of natural populations may be necessary to test the prediction thoroughly. Focusing upon transposition directly, the prediction is simply that transposition will be induced in progeny from a cross between two strains that differ significantly in the number of members within a particular family of middle-repetitive sequences. Transposition will be most frequent when the male parent of the original cross comes from the strain with the largest number of elements.

A correlation between mutation rate and transposition would show that transposable elements are not strictly selfish. They would express themselves through a major population-level phenotype, and their behaviour could be regulated by selection on that phenotype. Transposable middlerepetitive sequences may, in fact, be the principal agents of spontaneous mutation. The logical conclusion of this line of inquiry, though, is perhaps the most intriguing of all. When conditions determining the intracellular behaviour of these elements are better understood, it may be possible to predict, or even control, some part of the spontaneous mutation that is responsible for both the useful and the traumatic variation in all species.

Acknowledgements.-We are especially grateful to Michael Ashburner and Michael Young for hospitality in their labs and for discussing some of these ideas. We also thank Francis Crick, Ford Doolittle, and James Shapiro for their helpful comments on the manuscript. This work was supported by a NATO grant for international collaboration, by a Biomedical Sciences Support grant from the University of Oklahoma to J.N.T., and NSF grant DEB7923007 to R.C.W., who is the recipient of Research Career Development Award ES-00087 from NIEHS.

\section{REFERENCES}

ALEXANDER, M. L. 1949. Note on gene viability in natural populations of Drosophila. Univ. Texas Publ, , 4920, 63-69.

ANANIEV, E. V., GVOZDEV, V. A., ILYIN, YU. V., TCHURIKOV, N. A., AND GEORGIEV, G. P. 1978. Reiterated genes with varying location in intercalary heterochromatin regions of Drosophila melanogaster polytene chromosomes. Chruinosoma, 70, 1-17.

AUERBACH, C. 1976. Mutation Research. Chapman and Hall, London.

BERG, R. L. 1965. Studies of mutability in geographically isolated populations of Drosophila melanogaster Meig. Mutation in Population, Symposium on the Mutation Process. Prague. pp. 61-74.

CAMERON, J. R., LOH, E. Y., AND DAVIS, R. W. 1979. Evidence for transposition of dispersed repetitive DNA families in yeast. Cell, 16, 739-751.

COHEN, S. N., AND SHAPIRO, J. A. 1980. Transposable genetic elements. Sci. Amer., 242, 36-45.

CRICK, F. H. C. 1979. Split genes and RNA splicing. Science, 204, 264-271.

DAWKINS, R. 1976. The Selfish Gene. Oxford University Press, London.

DOOlitTlE, W. F., AND SAPIENZA, C. 1980. Selfish genes, the phenotype paradigm, and genome evolution. Nature, 284, 601-603.

Dover, G., AND DOolitTle, W. F. 1980. Modes of genome evolution. Nature, 288, $646-647$.

ENGELS, W. R. 1979. Extrachromosomal control of mutability in Drosophila melanogaster. Proc. Natl. Acad. Sci., U.S.A., 76, 4011-4015.

FARABAUGH, P. J., AND FINK, G. R. 1980. Insertion of the eukaryotic transposable element Ty1 creates a 5-base pair duplication. Nature, 286, 352-356.

GEHRING, W. J., AND PARO, R. 1980. Isolation of a hybrid plasmid with homologous sequences to a transposing element of Drosophila melanogaster. Cell, 19, 897-904.

GOLUBOVSKY, M. D., IVANOV, YU. N., AND GREEN, M. M. 1977. Genetic instability in Drosophila melanogaster: Putative multiple insertion mutants at the singed bristle locus. Proc. Natl. Acad. Sci., U.S.A., 74, 2973-2975. 
GREEN, M. M. 1978. The genetic control of mutation in Drosophila. Stadler Symposium, 10, 95-104.

GREEN, M. M., AND SHEPHERD, S. H. Y. 1979. Genetic instability in Drosophila melanogaster: The induction of specific chromosome 2 deletions by $M R$ elements. Genetics, 92, 823-832.

HEFFRON, F., MCCARTHY, B. J., OHTSUBO, H., AND OHTSUBO, E. 1979. DNA sequence analysis of the transposon $\operatorname{Tn} 3$ : Three genes and three sites involved in transposition of Tn3. Cell, 18, 1153-1163.

HENDERSON, S. A., WOODRUFF, R. C., AND THOMPSON, J. N., JR. 1978. Spontaneous chromosome breakage at male meiosis associated with male recombination in Drosophila melanogaster. Genetics, 88, 93-107.

ISING, G., AND RAMEL, C. 1976. The behaviour of a transposing element in Drosophila melanogaster. In The Genetics and Biology of Drosophila, eds. M. Ashburner and E. Novitski. Vol. 1b, pp. 947-954. Academic Press, London.

IVES, P. T. 1950. The importance of mutation rate genes in evolution. Evolution, 4, 236-252.

KIDWELL, M. G., KIDWELL, J. F., AND SVED, J. A. 1977. Hybrid dysgenesis in Drosophila melanogaster: a syndrome of aberrant traits including mutation, sterility and male recombination. Genetics, 86, 813-833.

LEIGH, E. G., JR. 1973. The evolution of mutation rates. Genetics (Suppl.), 73, 1-18.

MCCLINTOCK, B. 1952. Chromosome organization and genic expression. Cold Spring Harbor Symp. Quant. Biol., 16, 13-47.

MIRZABEKoV, A. D. 1981. Mobile genetic elements: new Soviet Studies. Nature, 291, 12. ORGEL, L. E., AND CRICK, F. H. C. 1980. Selfish DNA: the ultimate parasite. Nature, 284, 604-607.

ORGEL, L. E., CRICK, F. H. C., AND SAPIENZA, C. 1980. Selfish DNA. Nature, 288, 645-646. POTTER, S. S., BROREIN, W. J., JR., DUNSMUIR, P., AND RUBIN, G. M. 1979. Transposition of elements of the 412, copia, and 297 dispersed repeated gene families in Drosophila. Cell, 17, 415-427.

POTTER, S., TRUETT, M., PHILliPS, M., AND MAHER, A. 1980. Eukaryotic transposable genetic elements with inverted terminal repeats. Cell, 20, 639-647.

ROEDER, G. S., FARABAUgh, P. J., CHALEFF, D. T., AND FINK, G. R. 1980. The origins of gene instability in yeast. Science, 209, 1375-1380.

SHAPIRO, J. A. 1979. Molecular model for the transposition and replication of bacteriophage Mu and other transposable elements. Proc. Natl. Acad. Sci., U.S.A., 76, 1933-1937.

SLATKO, B. 1978. Evidence for newly induced genetic activity responsible for male recombination induced in Drosophila melanogaster. Genetics, 90, 105-124.

SPENCER, W. P. 1935. The non-random nature of visible mutations in Drosophila. Amer. Naturalist, 69, 223-238.

STROBEL, E., DUNSMUIR, P., AND RUBIN, G. M. 1979. Polymorphisms in the chromosomal locations of elements of the 412, copia, and 297 dispersed repeated gene families in Drosophila. Cell, 17, 429-439.

STURTEVANT, A. H. 1937. Essays on evolution. I. On the effects of selection on mutation rate. Quart. Rev. Biol., 12, 464-467.

THOMPSON, J. N., JR., AND WOODRUFF, R. C. 1978a. Mutator genes: Pacemakers of evolution. Nature, 274, 317-321.

THOMPSON, J. N., JR., AND WOODRUFF, R. C. 1978 $b$. Chromosome breakage: A possible mechanism for diverse genetic events in outbred populations. Heredity, 40, 153-157.

THOMPSON, J. N., JR., AND WOODRUFF, R. C. 1980. Increased mutation in crosses between geographically separated strains of Drosophila melanogaster. Proc. Natl. Acad. Sci., U.S.A., 77, 1059-1062.

VOELKER, R. A. 1974. The genetics and cytology of a mutator factor in Drosophila melanogaster. Mutation Res., 22, 265-276.

Williams, G. C. 1966. Adaptation and Natural Selection. Princeton University Press, Princeton, N.J.

WILliAMSON, V. M., YOUNG, E. T., AND CIRIACY, M. 1981. Transposable elements associated with constitutive expression of yeast alcohol dehydrogenase II. Cell, 23, 605-614.

WOODRUFF, R. C., AND THOMPSON, J. N., JR. 1980. Hybrid release of mutator activity and the genetic structure of natural populations. Evolutionary Biology, 12, 129-162.

WOODRUFF, R. C., THOMPSON, J. N., JR., AND LYMAN, R. F. 1979. Intraspecific hybridisation and the release of mutator activity. Nature, 278, 277-279.

WOODRUFF, R. C., SLATKO, B. E., AND THOMPSON, J. N., JR. 1982. Factors affecting mutation rate in natural populations. In The Genetics and Biology of Drosophila, eds. M. Ashburner, H. L. Carson, and J. N. Thompson, jr. Vol. 3c, in press. Academic Press, London. 
YANNOPOULOS, G. 1978. Studies on male recombination in a Southern Greek Drosophila melanogaster population. (c) Chromosomal abnormalities at male meiosis. (d) Cytoplasmic factor responsible for the reciprocal cross effect. Genet. Res., Camb., 31, 187-196. YOUNG, M. W. 1979. Middle repetitive DNA: A fluid component of the Drosophila genome. Proc. Natl. Acad. Sci., U.S.A., 76, 6274-6278. 